\title{
El impacto ambiental de las actividades físico-deportivas en el medio natural. El caso de la práctica del Mountain Bike o bicicleta todo terreno The environmental impact of physical and sporting activities in the natural environment. The case of Mountain Bike
}

\author{
*Estela Inés Farías Torbidoni, **Oriol Sallent, \\ *Instituto Nacional de Educación Física de Catalunya (España), **Universitad de Vic (España)
}

Resumen: En mayor o menor medida, la práctica de cualquier tipo de actividad físico-deportiva en el medio natural puede provocar diferentes niveles de impacto sobre el medio natural en el que se desarrollan. Disponer de un nivel mínimo de conocimientos acerca de las características principales de estos impactos, elaborar las correspondientes recomendaciones prácticas sobre la forma en el que estos pueden ser minimizados y sobre todo, darlos a conocer, es una de las numerosas contribuciones que los profesionales de la actividad física y el deporte pueden aportar en torno a una práctica más sostenible de este tipo de actividades, y con esta, una mejor conservación del medio natural en el que se desarrollan. En el presente artículo, y tomando como punto de referencia uno de los documentos de trabajo elaborados durante el año 2007-2008 en el marco del Pla Verd de l'Esport, de la Generalitat de Catalunya (Generalitat de Catalunya, Departament de la Presidencia, Secretaría General de l'Esport), concretamente, en el relativo a la elaboración de una Guía de Buenas Prácticas para el caso de la práctica del Mountain Bike (MTB) o Bicicleta Todo Terreno (BTT), los autores de este artículo presentan, a modo de ejemplo, algunos de los principales aspectos metodológicos y prácticos considerados en la sistematización de la información disponible, siendo la valoración de los diferentes canales o alternativas de difusión existentes otros de los aspectos que se abordan en este sentido. Con ello se pretende ofrecer un nuevo enfoque que contribuya en la construcción de un diálogo objetivo y constructivo entre las diferentes actores o partes implicadas, ya sean estos gestores del territorio, federaciones deportivas, practicantes, etc.

Palabra clave: actividades físico-deportivas en el medio natural, aproximación metodológica, minimización impactos ambientales, Bicicleta Todo Terreno.

Abstract: Outdoor sport activities have an impact on the environment in a certain level. Sports professionals and practitioners can contribute to a more sustainable practice and better environment conservation by investigating about the main characteristics of these impacts, elaborate proper guidelines to minimize them and, over all, communicate this knowledge widely. In this article the authors propose a methodology to systematise all information available. This proposal arise from an initiative of the Catalan Government - Sports Board (Generalitat de Catalunya, Departament de la Presidencia, Secretaría General de l'Esport) in the frame of securing and improving the environmental practice of sports initiated in 2007 (Pla Verd de l'Esport), and more specifically, the designing and diffusion of a Guidelines for Mountain Bike booklet. To contribute to discussion of differents implicates parts: country management, sport federation between others.

Key words: oudoor activity, methodological approach, environment impact minimizing, mountain bike.

\section{Introducción}

Antes que nada, se ha de tener en cuenta, que un impacto ambiental aparece cuando una acción o actividad provoca una alteración en el medio natural o en algunos de los componentes del medio en el que se desarrolla (Hammitt y Cole, 1998); siempre teniendo en cuenta, que esta definición no siempre implica efectos negativos, sino que pueden ser, tal y como sucede en muchas ocasiones, beneficiosos para el entorno natural y social en el que se desarrollan.

En general, los impactos ambientales que pueden llegar a provocar este tipo de prácticas no dependen de un solo aspecto, como lo podría ser las características intrínsecas de la actividad practicada (movilidad, requerimientos técnicos, necesidades de equipamientos y/o vehículos), sino de un continuo de factores ya relacionados por Hammitt and Cole (1998) como: el medio natural en el cual se desarrollan (capacidad de acogida física, social y ecológica, plasticidad o capacidad de resiliencia del medio, existencia o no de especies protegidas, presencia de ecosistemas especialmente sensibles que pueden ser afectadas, etc.), las características de los practicantes que las practican (número de integrantes por grupo, tipología de los mismos, grado de concienciación y compromiso hacia el medio ambiente, otros aspectos conductuales etc.) o la forma en que estas son practicadas (intensidad, distribución temporal y espacial, grado de organización, nivel de implantación de la actividad en la zona o región, etc.).

Fecha recepción: 28-02-09 - Fecha envío revisores: 07-03-09 - Fecha de aceptación: 25-05-09 Correspondencia: Farías Torbidoni Estela

Consultoría Socioambiental Ecogestion.

Afores s/n (25154). Castelldans - Lleida

E-mail: estela.farias@cag.es
En este sentido son numerosos los trabajos, informes y/o publicaciones que tratan sobre los diferentes tipos de impactos ambientales que pueden ocasionar este tipo de actividades o modalidades deportivas. Así tenemos, por ejemplo, las que abordan de forma genérica, entre otros aspectos, una descripción de los principales impactos que pueden ser generados por un conjunto heterogéneo de actividades deportivas o recreativas, como los realizados por AEDENAT (1994), Andrés, Blanco, Pertejo, y Prats, M. (1995), Benayas (2000), Hammitt y Cole (1998), NPCA (1992a y b) o Villalvilla, Blázques y Sánchez (2000); como las que de una forma más específica han estudiado los impactos para un determinado tipo de actividad o actividades deportiva/s sobre un espacio, zona o ecosistema natural concreto: Andres-Abellan, Del Alamo, Landete-Castillejos, Lopez-Serrano, Garcia-Morote y Del Cerro-Barja (2005), Benayas, Blanco y Pruebe (1996); Gómez-Limón (1996), Lynn y Brown (2003), Thurston y Reader (2001), Wilson y Seney (1994). Sin dejar de considerar aquellos trabajos, que de alguna forma, han intentado fusionar estos dos grandes bloques temáticos: impactos ambientales genéricos y específicos y principales connotaciones prácticas para los diferentes grupos de actividades. Trabajos como los elaborados, a nivel internacional, por organizaciones como: Bundesamt für Naturschutz (Biedenkapp y Stührmann, 2004), la International Mountain Bicycling Association (IMBA 2004, 2007), Parcs Naturels Regionaux de France (Van Lierde, 2007), o a nivel nacional, por Ecotrans-España (Andrés et al. 1995), el Comité Olímpico Internacional (Tarradellas, 2005), o Green Cross-España (2008), son una buena muestra de ello.

Los estudios sobre los impactos ambientales provocados por la práctica de las diferentes modalidades deportivas en el medio natural tienen una gran importancia, no tan sólo por el hecho de poder considerarse como un instrumento clave en el diseño de las consideraciones ambientales oportunas (relativas tanto en la planificación como en la 
gestión y/o práctica de este tipo de actividades); sino también por la circunstancia de poder constituirse como un buen vehículo de comunicación entre los diferentes partes o actores implicados (léase: gestores del territorio, entidades deportivas, asociaciones ecologistas, practicantes, etc.).

Mediante la presentación de una propuesta de sistematización de los conocimientos o información disponible en torno a los impactos ambientales, en este caso, de la practica de la Bicicleta Todo Terreno (BTT) o Mountain Bike (MTB), y la discusión/valoración de los diferentes canales o alternativas existentes en relación a difusión de los conocimientos disponibles en este sentido, el presente artículo pretende contribuir en la construcción de un diálogo más objetivo y constructivo entre las diferentes partes o actores implicadas; intentando de esta forma hacer camino en la consecución de una práctica más sostenible de este tipo de actividades, y con esta, una mejor conservación del medio natural en el que se desarrollan.

\section{Metodología}

En términos generales, la metodología de trabajo utilizada en la sistematización de información disponible, tanto en torno a los impactos ambientales susceptibles de ser generados por la práctica de este tipo de este tipo de actividades, como en la forma de minimizar el volumen de los mismos, se basó en la consideración de las siguientes fases, y recopilación de los correspondientes indicadores:

-Fase 1: Sistematización de la información disponible a nivel divulgativo y científico en relación a dos grandes apartados: Impactos Ambientales Genéricos e Impactos Ambientales Clave o intrínsecos a la actividad

-Fase 2: Fundamentación de la selección de estos últimos (impactos claves) en base a la consulta y cita de las diferentes fuentes bibliográficas (estudios empíricos, descriptivos, informes, etc.): Identificación de los Aspectos a tener en cuenta, Datos de interés y Otros efectos a ser considerados

-Fase 3: Propuesta de un grupo reducido de indicadores, a modo de ejemplo, susceptibles de ser utilizados en la evaluación o seguimiento de estos impactos

-Fase 4: Elaboración de las correspondientes recomendaciones de práctica y de gestión

\section{Indicadores}

*Datos generales: en donde además de detallar el nombre específico de la actividad objeto de estudio, se especificarían las diferentes modalidades deportivas incluidas, como así también un breve descripción de la/s misma/s

*Impactos ambientales: apartado que en este caso y bajo un criterio claramente práctico, estaría desglosado en dos sub-apartados

-Impactos ambientales potenciales genéricos, a modo de información general que incluiría un detalle de los mismos, y que en muchos casos podría ser común a la práctica de un mismo grupo o conjunto de actividades físico-deportivas según corresponda (ejemplo, actividades de tierra, agua, aire)

-Impactos ambientales específicos, que como bien lo indica su denominación, resultan claves a la hora de valorar las repercusiones de la actividad en particular sobre el medio natural en el que se desarrollan

*Datos de interés y otros efectos: en donde además de incluir, en función de la información disponible, datos que avalen o justifiquen las repercusiones ambientales de la correspondiente tipología de impactos desarrolladas (genéricos o clave), incluiría determinados aspectos de la actividad, que de forma ciertamente indirecta, podrían concurrir en un incremento de algunos de estos impactos

*Indicadores de alarma y/o posibles medidas de observación y seguimiento: que además de ejemplificar la forma en el que estos impactos claves podrían ser identificados, plantearía, a modo de ejemplo, algunos indicadores visuales útiles a la hora de detectar o realizar un seguimiento de los correspondientes indicadores de alarma
*Recomendaciones de práctica y de gestión: que como bien lo indica su nombre, estaría orientado a la elaboración de las correspondientes actuaciones prácticas de planificación y dirección en base a la información disponible

*Bibliográfica y fuentes de consulta, en relación al detalle de las fuentes consultadas en la elaboración de los diferentes indicadores

\section{El caso de la práctica del mountain bike (MTB) o bicicleta} todo terreno (BTT)

A continuación se exponen, los principales aspectos considerados en cada uno de los diferentes indicadores propuestos, tomando como ejemplo o estudio de caso, la práctica del MTB, modalidad de travesía o marcha deportiva (léase; realización de cualquier tipo de excursión en BTT - que se lleva a cabo utilizando mayoritariamente pistas forestales, caminos de tierra existentes o senderos expresamente diseñados para la práctica de este u otro tipo de prácticas ciertamente compatibles excursionismo, etc. -). En este sentido destacar, que la descripción de los indicadores que a continuación se detallan, y con una clara intención de aunar criterios, ha sido elaborada obviando algunos aspectos de tipo más sociales como lo podrían ser las problemáticas asociadas al empleo de un mismo recurso por parte de diferentes modalidades deportivas.

\section{* Impactos ambientales genéricos.}

-Sobre la vegetación: disminución del crecimiento y diversidad de la cobertura vegetal, disminución sobre su capacidad reproductiva, alteración de la estructura de edades y cambios en la comunidades vegetales (las especies resistentes al impacto provocado por esta actividad se hacen más abundantes)

-Sobre el suelo: destrucción de la capa superficial de materia orgánica, alteración del horizonte edáfico, compactación del suelo, alteración de las características básicas del suelo (aireación, temperatura, fauna edáfica, textura, nutrientes), reducción de la capacidad de infiltración, aumento del agua superficial, erosión y repercusiones sobre el crecimiento dela vegetación

-Sobre el agua: incremento del nivel de turbidez, entrada de nuevos nutrientes, alteración de su composición

- -Sobrela fauna: disminución de la calidad de los hábitats, perturbación puntual de determinadas especies, modificación de su comportamiento habitual...

\section{Aspectos básicos a tener en cuenta}

- En términos generales siete son las principales fases que se pueden identificar en el proceso de erosión del suelo (reducción del horizonte orgánico de humus y hojarasca, descenso de la materia orgánica en el horizonte mineral inferior, compactación, perdida de la capacidad de filtración del agua, saturación y anoxia a nivel de la raíz, escorrentías superficiales, erosión del suelo), siendo las últimas fases las más criticas por su dificultad de irreversibilidad natural (Hammitt y Cole, 1998) - La compactación, la perdida de la cobertura vegetal y la disminución de la capacidad de infiltración provocan un aumento de la escorrentía iniciando el proceso de erosión. El agua se canaliza por los senderos que progresan en amplitud y profundidad, pudiendo llegar a modificar la escorrentía local y la red de drenaje de forma importante (Benayas, 2000; Gómez-Limón, 1996; Hammitt y Cole, 1998)

- En este sentido, se ha de tener en cuenta, que la resistencia natural del suelo disminuye de forma bastante importante con la humedad (Wilson y Seney, 1994)

\section{* Impactos ambientales clave o intrínsecos a la actividad.}

-Perdida de la cobertura vegetal. Sobre todo en el caso de circulación fuera de caminos o red principal de itinerarios...

-Proceso de erosión del suelo

\section{Datos de interés}

- Durante el primer año de la creación de un itinerario es cuando se genera el mayor volumen de impacto. Apartir de este primer año el nivel de impacto generalmente se mantiene, tardando en desaparecer si se deja de utilizar según el hábitat, entre cinco y quince años (IMBA, 2007; 
Leung y Marion, 1996; Marion, 2006). Cabe destacar en este sentido, la constatación por parte de algunos trabajos experimentales realizados al respecto por Thruston y Reader (2001) en relación a la existencia de una mayor concentración de este tipo de impactos (léase; exposición del suelo y pérdida de la densidad y diversidad de la vegetación) en la zona central del sendero ( $30 \mathrm{~cm}$ aproximadamente)

- El nivel de impacto generado por la práctica de esta actividad sobre la vegetación resulta severo a partir de una frecuencia de paso equivalente a 500 pasos anuales (número de veces que transita la bicicleta sobre un determinado punto del sendero a lo largo de un año natural), Cole (1990). En este sentido Thruston y Reader (2001) han demostrado que con este número de pasos el nivel de exposición del suelo puede llegar a incrementarse en un $49 \%$, llegando a ser la pérdida de vegetación generado en este proceso de prácticamente el $\mathbf{1 0 0 \%}$ - La pendiente y el alineamiento del itinerario (pendiente lateral) pueden ser considerados como indicadores que pueden predecir el nivel de impacto susceptible de ser producido por la práctica de esta actividad. Los itinerarios con una pendiente superior al $16 \%$ son más vulnerables a los procesos erosivos que los que albergan una pendiente inferior a este porcentaje (IMBA, 2007).

- La pérdida de suelo generada por la práctica de esta actividad puede llegar a ser tres veces inferior a la pérdida generada por el excursionismo (202 y 669 pies cúbicos/milla) y más aún si los comparamos con los provocados por la práctica de otro tipos de actividades como el excursionismo a caballo o del motociclismo en el medio natural (5,396 y 8,235 pies cúbicos/milla, respectivamente)

\section{* Otros efectos. \\ -Perturbación de la fauna en cuanto a la posible calidad de sus} hábitats

-Impactos sobre el paisaje y otros aspectos del medio: posible abandono de residuos, efectos derivados del impacto sobre el suelo o la vegetación en relación a la creación de trazados alternativos, paralelos o ampliaciones puntuales de los senderos principales, debido a un inadecuado uso de estos (circulación en paralelo, velocidad excesiva, creación de atajos, etc.)

-Efectos derivados de una falta de planificación, adecuación o uso de zonas de acceso utilizados generalmente para el aparcamiento de vehículos; sobre todo en puntos ciertamente masificados. Aparición de posibles impactos visuales negativos como consecuencia de una excesiva o inadecuada señalización de los itinerarios o red de itinerarios utilizados.

\section{* Indicadores de alarma.}

-Aparición de atajos, trialeras o ampliaciones puntuales del trazado principal

-Aparición de cárcavas donde la pérdida de suelo supera los $10 \mathrm{~cm}$ de profundidad.

-Aparición de residuos al inicio, final, o a lo largo del recorrido

\section{Posibles indicadores de medida o seguimiento}

- Porcentaje lineal de senderos alternativos/atajos o trialeras (longitud total itinerario x 100 / total metros lineales de senderos alternativos observados)

- Número de puntos conflictivos o tramos de itinerarios con evidentes niveles de erosión. Longitud total itinerario/número de puntos conflictivos detectados

- Número de residuos o puntos de acumulación de residuos detectados a lo largo de un número representativo de tramos dentro del itinerario o red de itinerario considerado. Longitud total itinerario/número de puntos con acumulación de residuos detectados

\section{* Recomendaciones específicas para los practicantes.}

-Durante el primer año de la creación de un itinerario es cuando se genera el mayor volumen de impacto (IMBA, 2007; Leung y Marion 1996; Marion, 2006). De aquí la importancia de evitar al máximo la creación de senderos alternativos y de respetar el trazado principal de los diferentes itinerarios.
- Algunas publicaciones como las realizadas por IMBA (2004, 2007) destacan la importancia que puede tener la forma en el que se conduce la bicicleta sobre el nivel de impacto que se puede provocar (velocidad, frenadas, ángulos de giro, derrapadas). Las pendientes utilizadas para el descenso son susceptibles de ser más erosionadas (Goeft y Alder, 2001). La humedad y la pendiente del suelo puede incidir de forma exponencial sobre el nivel de erosión provocado por la práctica de esta actividad (IMBA 2007). Resulta imprescindible valorar en profundidad estos aspectos (velocidad, sentido de la marcha, grado de humedad del terreno, etc.) a la hora de practicar esta actividad, y sobre todo, en senderos situados en zonas especialmente frágiles o próximas a espacios naturales protegidos.

-Teniendo en cuenta que el tamaño del grupo puede incidir de forma negativa sobre el nivel de impactos susceptibles de ser provocados por la práctica de esta actividad... Intentar, en el momento de practicar esta actividad en grupos numerosos (más de 6 personas), circular de forma ordenada, no hablar demasiado fuerte y ser especialmente respetuoso con el entorno que los rodea. Valorando siempre la opción menos impactante a la hora de elegir el o los itinerarios a recorrer (temporada del año en relación a la humedad, zonas especialmente frágiles que ostenten alguna figura de protección, épocas de reproducción de determinadas especies, etc.)..

* Recomendaciones generales para las administraciones responsables.

-En términos generales cinco son los principales factores identificados por la literatura científica existente, en relación al nivel de impacto ambiental generado sobre el suelo por la práctica de esta actividad: tipo de suelo, pendiente, alineamiento del trazado principal, ángulos de giro, grado de humedad y nivel de mantenimiento del sendero si corresponde (Goeft, 2000; IMBA, 2007; Marion, 2006; White, Waskey, Brodehl y Foti, 2006; Wilson y Seney, 1994). Considerar estos aspectos a la hora de planificar o gestionar la oferta de itinerarios existentes en este sentido es una buena forma de prevenir o minimizar el nivel de impacto susceptible de ser generado por la práctica de esta actividad.

-La capacidad de resiliencia (habilidad de retornar al su estado inicial) de la vegetación afectada en relación a la practica del MTB se incrementa en el momento que la actividad no se practica de forma continua (Cole, 1990), por lo que el cierre o uso intermitente de determinados itinerarios pueden ser una buena medida en la minimización de los impactos ambientales negativos producidos sobre la vegetación. Realizar una correcta planificación en relación a la oferta de itinerarios de una determinada zona o región, procurando evitar la masificación y el uso continuo de un mismo itinerario o circuito, se constituye como una buena estrategia de planificación/gestión en este sentido.

-Son numerosos los estudios que destacan la importancia que tiene la realización de una buena planificación del sistema o red de itinerarios, ya sea esta a nivel local, regional, etc. Cuando una red no esta planificada se incrementa la creación de senderos alternativos y la aparición de posibles conflictos entre los diferentes usos (IMBA 2004). Una buena conectividad de la red de itinerarios contribuye muy positivamente en la disminución del impacto sobre el suelo y la vegetación susceptible de ser provocado por la práctica de esta y otras modalidades deportivas (Hammitt y Cole, 1998). Resulta imprescindible realizar un esfuerzo en cuanto a la planificación del sistema o red de itinerarios, intentando siempre que sea posible, incluir en este esfuerzo a los principales actores implicados (practicantes) sensibilizándolos de la importancia de su comportamiento individual...

-Así mismo, autores como Hammitt y Cole (1998), ponen de manifiesto la incidencia que puede tener sobre el nivel de impacto susceptible de ser provocado por la practica de esta actividad, no tan sólo la fragilidad, durabilidad o capacidad de resiliencia del medio, sino otros aspectos de tipos más sociales como la cantidad y distribución del uso (frecuentación recreativa), número de integrantes y composición social de los practicantes que componen el grupo, comportamiento, experiencia, motivaciones y/o conocimientos sobre el mínimo impacto. Profundizar en el conocimiento de las características principales de los practi- 
cantes de esta actividad, en determinadas zonas o áreas con un cierto volumen de uso, resulta de fundamental importancia (para más información consultar en bibliografía general Farías, 2000, 2002 y Farías y Tolosa, 2004.).

\section{Algunos ejemplos medidas preventivas}

- Delimitar en la mayor medida posible el trazado del itinerario principal disuadiendo el uso o creación de senderos alternativos... (Colocando troncos, piedras, señalizaciones específicas, etc.)

- Tratar con especial atención las características de drenaje de los mismos. Valorando en profundidad el diseño o la gestión (mantenimiento) de los diferentes itinerarios en relación a la pendiente y ángulos de giro incluidos en los diferentes tramos

- Realizar un correcto mantenimiento de los tramos de itinerarios intentando minimizar su amplitud con tal de evitar los impactos y la velocidad de circulación

- Evitar que el trazado de la oferta de itinerarios pase por zonas de elevada o crítica sensibilidad ambiental (hábitats, fauna, etc.)

- Educar y sensibilizar a los practicantes en el mínimo impacto mediante la elaboración de diferentes programas de información, educación o sensibilización (residuos, comportamiento apropiado, etc.)

- Implicar a los practicantes en el diseño de recorridos, en las medidas reguladoras y en los mensajes de sensibilización

- Señalizar adecuadamente en el caso que corresponda (en función de la normativa existente o conocimientos científicos o técnicos pertinentes) las épocas del año más adecuadas para el uso de los itinerarios en cuestión

\section{Algunos ejemplos medidas correctoras}

- Intentar modificar el trazado cuando sea posible evitando aquellas zonas susceptibles por sus características a un mayor impacto ambiental (pendiente, tipo de suelo, etc.)

- Poner en marcha medidas correctoras en aquellos puntos conflictivos degradados o potencialmente degradables como canaleras de agua, obstáculos para reducir la velocidad, etc.

- Potenciar el uso de aquellos itinerarios con una funcionalidad y diseño acordes a los aspectos anteriormente referenciados

- Instaurar un sistema de limpieza y de mantenimiento periódico y rotativo sobre aquellos itinerarios que presenten una mayor tendencia de conflictividad en relación a algunos de los impactos clave (presencia de cárcavas, etc.)

* Recomendaciones específicas en el caso de la organización de eventos deportivos.

- Los itinerarios o tramos que se habiliten expresamente para la realización de cualquier tipo de evento deportivo pasaran a formar parte de la red de itinerarios existentes en la zona, por lo que se ha de intentar:

- Evitar la creación de nuevos tramos de itinerarios

- Evitar el paso de los mismos en zonas protegidas o especialmente frágiles

- Planificar la obertura de estos bajo criterios preventivos a medio y largoplazo...

- Una correcta planificación y gestión de los accesos necesarios y de la presencia de público asistente al evento es una buena forma de minimizar los impactos potenciales de este tipo de eventos. Por lo que en lo posible habilitar y potenciar la utilización de transportes públicos, señalizar y delimitar correctamente las zonas de aparcamiento, contemplando un posible sistema de evacuación, etc.

- Restaurar, regenerar y limpiar el área en donde se ha desarrollado el evento se constituye como uno de los elementos claves de la gestión sostenible de este tipo de acontecimientos. Instaurar medidas técnicas que minimicen la erosión del suelo en aquellos puntos especialmente degradados (con la colocación de redes de contención, etc.), prever una gestión integral de los residuos que se puedan generar durante el evento (ej. racionalizar el consumo de los mismos, evitar los productor de «usar y tirar», implementar un sistema de recogida selectiva en los avituallamientos, etc.) y/o utilizar en la señalización de los mismos materiales biodegradables (pinturas, cintas, etc.), es una buena forma de disminuir los trabajos necesarios en este sentido. Al respecto es reco- mendable, siempre que sea posible, realizar el correspondiente Informe deAfecciones Ambientales de forma previa a la realización del evento. $\mathrm{Y}$ en todo caso, siempre intentar valorar de forma anticipada las medidas preventivas, correctoras y sistemas de control necesarios bajo el principio de precaución...

\section{Discusión}

En términos generales, nuestra experiencia en relación a la metodología que aquí se propone es aplicable a cualquier tipo de práctica físicodeportiva en el medio natural; pudiéndose, en este sentido, incluir otros tipos de indicadores en el caso que las prácticas o los agentes receptores así lo requieran (ej. principales grupos de interés, iniciativas en marcha, etc.). En este sentido se ha de tener en cuenta, que los indicadores aquí propuestos, fueron ya utilizados por los autores de este artículo en la recopilación y sistematización de la información disponible en torno a prácticas tan dispares como, el piragüismo, la escalada en roca, el excursionismo a pie, a caballo o la práctica del trial o moto enduro.

La variedad y el nivel de profundidad con el que han sido tratados los indicadores aquí propuestos posibilitan adecuar el contenido de cada uno de ellos a la utilización de diferentes canales de información, en donde la complejidad de los datos e información suministrada estaría supeditada a las características particulares de los diferentes actores o destinatarios implicados.

A medida que se analizan los posibles canales de información susceptibles de ser utilizados en la difusión de la información aquí sistematizada, se hace necesario recurrir a un análisis en profundidad de las principales partes o actores implicados. Conocer el nivel de sensibilidad o de conocimientos disponibles en torno a la problemática, o el nivel de especialización de los diferentes grupos de practicantes (principiantes, aficionados, profesionales, etc.), podría contribuir a una mejor selección, tanto de los contenidos como de los canales de difusión más apropiados.

La búsqueda de un adecuado equilibrio entre el nivel de conocimientos necesarios, la optimización de los recursos disponibles en la difusión de los mismos, y sobre todo, la selección de los canales más apropiados, se constituyen como algunos de los pilares fundamentales a ser considerados en el diseño de cualquier tipo de campaña de sensibilización; previa consideración, claro está, de la importancia que la participación o autogestión de las partes implicadas juega en este sentido.

Un ejemplo al respecto podría ser la elaboración consensuada por parte de los principales actores implicados de una pequeña ficha que contemple algunos de los aspectos y recomendaciones básicas como las planteadas a lo largo de la presentación de este artículo (léase, por ejemplo, impactos claves, datos de interés, principales recomendaciones); ya sea, a un nivel más organizativo, en la edición de una pequeña Guía de Buenas Prácticas relativa a la organización de este tipo de eventos (léase, marchas populares, competitivas, etc.), o a nivel más práctico, en el uso de los recursos naturales implicados en un territorio determinado. Siempre teniendo en cuenta, que la garantía de la eficacia de este tipo de guías, y en palabras de Wolf y Appel-Kummer, (2004), ha de basarse en el hecho de que las recomendaciones que en estas se detallen sean aceptadas y asumidas por todas las partes implicadas (sean estos gestores, practicantes o población local del entorno natural en cuestión).

En este sentido, y por muchas campañas de sensibilización y control que se implanten, la autogestión del practicante se constituye no solo como una de las herramientas más eficaces, sino también como una de las más duraderas en el tiempo. Las acciones consensuadas tienden a llegar mejor a buen puerto, como así también las acciones basadas en buenos fundamentos tienen más fuerza a la hora de ser defendidas. Generar procesos de participación y autoorganización, según el territorio y las inquietudes, es una buena manera de materializar este hecho, aunque no siempre sea posible.

Se trata de plantear un nuevo enfoque a la resolución de los problemas ambientales generables por la práctica de este tipo de actividades, que englobe aspectos científicos como los descritos en este artículo, 
pero también emocionales y políticos. Así, por ejemplo, se está trabajando desde la federación catalana de ciclismo, que lidera la lucha por evitar la prohibición de espacios de práctica, que utilizando la información obtenida a travéz de la metodología aquí descrita, y con la colaboración de la Secretaria General de l'Esport, ha iniciado una campaña de concienciación de los practicantes, mientras vehicula diferentes canales de diálogo con administraciones medioambientales para gestionar con criterio y sentido común la práctica de este tipo de actividades; en lo que es un largo camino que hace tiempo que se tendría que haber empezado a caminar.

Es nuestra voluntad, que el ejercicio de sistematización de los impactos medioambientales aquí presentado, lejos de constituirse como un mero ejercicio intelectual, sirva referencia en este camino como elemento de concienciación, integración, implicación y participación conjunta de los diferentes actores o partes implicados. Para ello, hay que ser cautos, y reconocer la dificultad de generalizar y transferir los resultados de los estudios aquí presentados a determinados entornos ambientales. Razón, por la que estos estudios, han de ser utilizados como complemento a una concertación, que solo será posible, con dos actitudes firmes: la gestión del territorio, de los usos y los recursos basados en criterios objetivos y aplicaciones prácticas abordadas según cada caso, y la voluntad de entendimiento y comprensión mutua a partir del conocimiento y respeto de la visión de la otra parte.

\section{Bibliografía}

Andres-Abellan, M., Del Alamo, J.B., Landete-Castillejos, T., LopezSerrano, F.R., Garcia-Morote, F.A. y Del Cerro-Barja, A. (2005) Impacts of visitors on soil and vegetation of the recreational area «Nacimiento del Rio Mundo» (Castilla-La Mancha, Spain). Environmental Monitoring And Assessment, 101(1-3), 55-67.

AEDENAT. (1994). El impacto de las actividades deportivas y de ocio/ recreación en la Naturaleza. València: AEDENAT.

Andrés, A., Blanco, R., Pertejo, J. y Prats, M. (1995). Manual para la mejora de la calidad ambiental de las actividades recreativas en la naturaleza. Madrid: ECOTRANS-España. Secretaría General de Turismo.

Biedenkapp, A. y Stührmann, E. (2004). Tourismus, Naturschutz und Wassersport. Skripten, 113.

Benayas, J.; Blanco, R. y Priebe, C. (1996). Análisis de los impactos ocasionados por el barranquismo en el Parque de la Sierra y los Cañones de Guara. Propuesta de Regulación. Servicio de Espacios Naturales Protegidos, Caza y Pesca. Aragón: Departamento deAgricultura y Medio Ambiente. Diputación General de Aragón. Tomo I, II, III

Benayas, J. (Coord). (2000). Manual de buenas prácticas del monitor de naturaleza: espacios naturales protegidos de Andalucía. Andalucía: Consejería de medio ambiente. Junta de Andalucía.

Cole, D.N. (1990). Ecological impacts of wilderness recreation and their management. En Hendee, Stankey y Lucas. Wilderness Management. Colorado: Editorial. Golden. 425-462.

Delcourt, G. (1999). Etudier la Fréquentation dans les Espaces Naturels - Méthodologie. Paris: ATEN.

Farías, E.I. (2000). El aprovechamiento recreativo, deportivo y turístico de los espacios naturales protegidos. Modelos de Frecuentación. El caso del Parc Nacional d'Aigüestortes i Estany de Sant Maurici. Tesis Doctoral. Institut Nacional d'Educació Física de Catalunya. Universitat de Lleida.

Farías, E.I. (2002). L'enquesta com a eina d'estudi per a la gestió recreativa del medi natural. Revista del Centre de Biodiversitat Habitats, 5, 16-27.

Farías, E.I. y Tolosa, G (2004). Una aproximació metodològica en la diagnosi socio-ambiental de l'ús recreatiu, esportiu i turístic dels espais naturals protegits: el cas de la Reserva del Congost de Montrebei. Butlletí de la Institució Catalana d'Història Natural, 72, 139153.
GREEN CROSS (2008) Guia de medio ambiente y sostenibilidad aplicados a los deportes no olímpicos. Barcelona: Fundació Barcelona Olímpica y Fundació Ernest Lluch.

Goeft, U. (2000). Managing mountain biking in Western Australia. Australian Parks and Leisure, 3, 29-31.

Goeft, U. y Alder, J. (2001). Sustainable mountain biking: a case study from the Southwest of Western Australia. Journal of Sustainable tourism, 9, 3-19.

Gómez-Limón, F.J. (1996). Uso Recreativo de los Espacios Naturales. Frecuentación, factores explicativos e impactos asociados. El caso de la comunidad de Madrid. Tesis Doctoral. Facultad de Ciencias. Departamento Interuniversitario de Ecología. Universidad Autónoma de Madrid.

Hammitt, W.E. y Cole, D.N. (1998). Wildland recreation. Canada: Ecology and management.

IMBA. (2004). Trail solutions: IMBA'S guide to building sweet singletrack. Boulder: International Mountain Bicycling Association.

IMBA. (2007). Managing mountain biking. IMBA'S guide to providing great riding. Boulder: International Mountain Bicycling Association.

Leung, Y.F. y Marion, J.L. (1996). Trail degradation as influenced by environmental factors: a state-of-the-knowledge review. Journal of soil and water conservation 51 (2), 130-136.

Leung, Y.F. y Marion, J.L. (2000). Recreation Impacts and Management in Wilderness: AState-of-Knowledge Review. USDAForest Service Proceedings RMRS-P-15, 5, 23-48

Lynn, N.A. y Brown, R.D. (2003). Effects of recreational use impacts on hiking experiences in natural areas. Landscape and Urban Planning, 64, 77-87.

Marion, J.L. (2006). Assessing and understanding trail degradation: results from Big South Fork National River and Recreational Area. USDA: National Park Service

NPCA (National Parks Conservation Association). (1992a). Visitor Impact Management. A review of research. Volume one. USDA: National Park Service.

NPCA (National Parks Conservation Association). (1992b). Visitor Impact Management. The planning. Volume two. USDA: National Park Service

Tarradellas, J. (2000). Guia sobre el deporte, el medio ambiente y el desarrollo sostenible. Madrid: Comité Olímpico Internacional.

Thurston, E. y Reader, R.J. (2001). Impacts of experimentally applied mountain biking and hiking on vegetation and soil of a deciduous forest. Environmental Management 27, 397-409.

Van Lierde, N. (2007). Sports de nature, outils pratiques pour leur gestion. Paris: L’Atelier.

Villalvilla, H., Blázquez, A y Sánchez, J. (2000). Deporte y naturaleza. El impacto de las actividades deportivas y de ocio en el medio natural. Madrid: Talasa.

Wilson, J.P. y Seney, J.P. (1994). Erosional impact of hikers, horses, motorcycles, and off-road bicycles on mountain trail in Montana. Mountain Research and Development, 14, 77-88.

White, D.D., Waskey, M.T., Brodehl, G.P y Foti, P.E. (2006). A comparative study of impacts to mountain bike trails in five common ecological regions of the south-western U.S. Journal of Park and Recreation Administration 24 (2), 21-41.

Wolf, A. y Appel-kummer, E. (2004). Freiwillige Vereinbarung Naturschutz - Natursport. Bundesamt fur Naturschutz Skripten, 106.

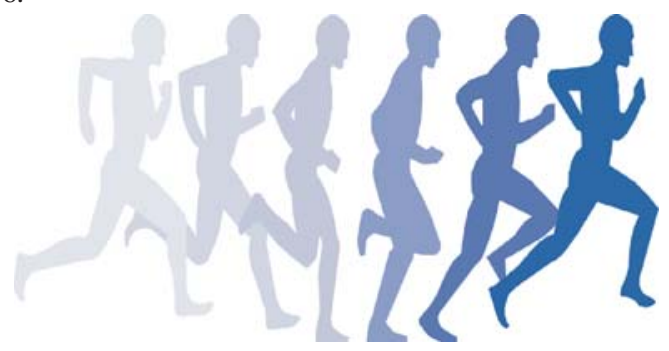

